\title{
Spectral Efficiency of Carbon Nanotube Antenna Based MIMO Systems in the Terahertz Band
}

\author{
Zheng Xu, Xiaodai Dong, Senior Member, IEEE, and Jens Bornemann, Fellow, IEEE
}

\begin{abstract}
Based on the models of single walled carbon nanotubes (SWCNTs) and bundled SWCNTs antennas, we analyse the spectral efficiency of an SWCNT bundled dipole antenna based MIMO system in the Terahertz band. Two scenarios are considered: the large scale MIMO and the conventional scale MIMO. It is found that, in order to get the maximum spectral efficiency, the CNT bundle size should be optimized to obtain a tradeoff between antenna efficiency and the number of antennas for a given area. This work also takes into account the random fluctuation in the bundle size during the CNT bundled antenna fabrication which reduces the system spectral efficiency.
\end{abstract}

Index Terms-Carbon nanotube antenna, MIMO networks, Terahertz, spectral efficiency.

\section{INTRODUCTION}

$\mathbf{T}$ HE multiple-input multiple-output (MIMO) antenna technique is well known to increase the spectral efficiency of a wireless communications system significantly. When used in a multiple user scenario, conventional MIMO design requires complex processing to mitigate the interuser interference. To deal with this problem, Marzetta et al. proposed a new kind of MIMO system with very large antenna arrays at the base station [1], [2]. However, practically implementing hundreds of antennas on one receiver poses serious size challenges in the Gigahertz $(\mathrm{GHz})$ band. At higher frequency bands in the Terahertz $(\mathrm{THz})$ range, the antenna length is scaled down to several hundred micrometers [3], and hence large scale MIMO concepts are well suited for use in these systems. In the micro- and nano-world of communications, new materials other than traditional metallic antennas may be explored for their unique characteristics and potential benefits.

As one of the novel materials, the single walled carbon nanotube (SWCNT) has attracted much interest for use in $\mathrm{THz}$ communication devices [4]. Reference [5] investigates the unique fundamental properties of SWCNTs. Based on these properties, some papers show the possibility to use SWCNTs as antennas [6], [7]. The slow wave propagation along SWCNTs can reduce the size of an antenna significantly. Compared with classical metallic antennas, the SWCNT antenna is more suitable for nanocommunications as its size is about one hundred times smaller. Burke et al. made some quantitative assessments of SWCNT's performance potential [8], [9]. It is pointed out that a SWCNT has conductivity several times larger than copper but has a very high resistance

Manuscript received May 18, 2013. The associate editor coordinating the review of this letter and approving it for publication was A. Bletsas.

The authors are with the Department of Electrical and Computer Engineering, University of Victoria, Victoria, BC, Canada, V8W 3P6 (e-mail: \{zhengxu, xdong, jbornema\}@ece.uvic.ca).

Digital Object Identifier 10.1109/WCL.2013.090313.130366 because of its small diameter. Due to the high resistance, the radiation efficiency of a SWCNT antenna is extremely low, which hampers its practical use. One approach to solve the resistance problem is to bind several SWCNTs as a bundle. Paper [10] studies the electrical properties of bundled CNTs, and [11] gives the performance prediction of SWCNT bundled dipole antennas. They showed that the antenna efficiency can increase by $30-40 \mathrm{~dB}$ when the bundle size is in the order of several hundreds.

Most of the existing works investigate the properties and performance of the SWCNT as a single dipole antenna. However, the performance of MIMO networks based on SWCNT bundled antennas has not been well studied yet. In this work, we analyse the uplink spectral efficiency of such MIMO network in two scenarios: large scale MIMO and conventional scale MIMO. In both scenarios, the efficiency of antennas and the number of antennas in a given receiving array area are controlled by the SWCNT bundle size. Through simulations, we determine the optimal bundle size that makes the spectral efficiency largest. Since it is hard to control the exact bundle size in real fabrication, the effect of fabrication variation is also demonstrated in this work. For comparison, we analyse the spectral efficiency of copper antennas based MIMO systems. Simulation results show that the proposed SWCNT antennas based system has a higher spectral efficiency than the conventional metallic antennas based system.

\section{MOdEL OF SWCNT AND SWCNT BUNDLED ANTENNA}

The SWCNT can be considered as a rolled up tube made of a graphene sheet which is formed by single layer carbon atoms. A circuit model of a SWCNT is shown in Fig. 1(a) [8], [10].

Ignoring the resistance due to impurities and defects, the intrinsic resistance of a single SWCNT is given by [9]

$$
R_{c n t}=\frac{h}{4 e^{2}} \frac{L}{l_{m f p}},
$$

where $L$ is the length of the SWCNT, $h$ is the Planck constant, $e$ is the electron charge, and $l_{m f p}$ is the mean free path in the SWCNT. $R_{c}$ in Fig. 1(a) is the SWCNT contact resistance which can be ignored when $L$ is large. Many experiments show that it is reasonable to take $l_{m f p}=1 \mu \mathrm{m}$ and $R_{c n t}=$ $6 \mathrm{k} \Omega / \mu \mathrm{m}$ [9]. As shown in previous work [12], the quantum capacitance is $C_{Q}=100 \mathrm{aF} / \mu \mathrm{m}$, the electrostatic capacitance $C_{E}$ is on the same order as $C_{Q}$, and the kinetic inductance is $L_{k}=16 \mathrm{nH} / \mu \mathrm{m}$. In this letter, we mainly focus on very narrow SWCNTs which have four parallel channels: two spinup channels and two spin-down channels. As the diameter of a SWCNT increases, the number of channels increases, and hence the performance study of thick SWCNTs needs to 


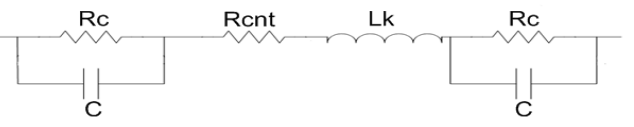

(a)

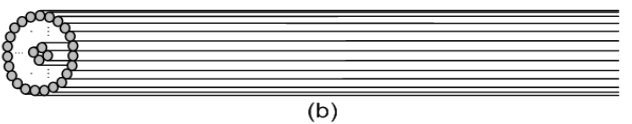

(b)

Fig. 1. (a) Circuit model of a single SWCNT [10]. (b) Several SWCNTs bound together as a bundle.

take into account the diameter of the SWCNT. For a very narrow SWCNT, the total capacitance $C$ is given by $C^{-1}=$ $C_{E}^{-1}+4 C_{Q}^{-1}$. The wave propagation speed in a SWCNT is

$$
v_{s}=\frac{1}{\sqrt{\frac{L_{k} C}{4}+\sqrt{\frac{L_{k}^{2} C^{2}}{16}+\frac{C^{2} R_{c n t}^{2}}{(2 \pi f)^{2}}}}},
$$

where $f$ denotes frequency. When the frequency is high enough (i.e. $1 \mathrm{THz}$ ), $v_{s}$ can be approximated as

$$
v_{s} \approx \sqrt{2 /\left(L_{k} C\right)}
$$

With the $L_{k}$ and $C$ values given above, $v_{s}=1.26 \times 10^{6}$ $\mathrm{m} / \mathrm{s}$, which is much smaller than the speed of light in free space. Due to the low transmission speed, the wave number $k_{p}=2 \pi / \lambda_{p}=2 \pi f / v_{s}$ on SWCNT antennas is much larger than that on conventional metallic antennas of the same frequency; $\lambda_{p}$ is the wavelength of the current distribution on a SWCNT. This makes the radiation resistance of SWCNT dipole antennas quite low [8]

$$
R_{r}=\left(k_{0} / k_{p}\right)^{2} \eta \beta\left(k_{p}, L_{a}\right),
$$

where $k_{0}$ is the wave number in free space, $\eta=120 \pi$ is the impedance of free space and function $\beta\left(k_{p}, L_{a}\right)$ is defined as $\beta\left(k_{p}, L_{a}\right)=\frac{1}{2 \pi} \int_{0}^{\pi} \sin ^{3} \theta\left[\frac{\cos \left(\frac{k_{0} L_{a}}{2} \cos \theta\right)-\cos \left(\frac{k_{p} L_{a}}{2}\right)}{1-\left(k_{0} / k_{p}\right)^{2} \cos ^{2} \theta}\right]^{2} d \theta$,

where $L_{a}$ is the length of antennas, and $\theta$ is the radiation direction. For $L_{a}=\lambda_{p}$, the resistance is about $5.7 \times 10^{-3} \Omega$. As a consequence, the radiation efficiency can be below -60 $\mathrm{dB}$ [8]. The radiation efficiency is expressed as

$$
\xi=P_{r} /\left(P_{r}+P_{o}\right)=R_{r} /\left(R_{r}+R_{o}\right),
$$

where $P_{o}$ is the ohmic loss power, $P_{r}$ is the radiation power, and $R_{o}=R_{\text {cnt }}$ is the ohmic resistance. Such low efficiency restricts the use of SWCNTs as antennas. One way to solve this problem is to bind several SWCNTs into a bundle. By doing this, the propagation speed $v_{s}$ increases while the ohmic resistance decreases. Reference [10] describes the properties of individual and bundled SWCNTs. Their measurements show that the impedance of the bundled SWCNT scales with the number of SWCNTs in the bundle. Paper [11] predicts the performance of SWCNT bundled dipole antennas as follows. Using the structure shown in Fig. 1(b), the resistance $R_{c n t}^{N}$ and kinetic inductance $L_{k}^{N}$ can be scaled down by the number of SWCNTs in the bundle while the capacitance $C$ remains unchanged [11]. At a high frequency (THz), $R_{c n t}^{N}, L_{k}^{N}, k_{p}^{N}$ and $\lambda_{p}^{N}$ can be written as $R_{c n t}^{N} \approx R_{c n t} / N, L_{k}^{N} \approx L_{k} / N$, $k_{p}^{N} \approx \sqrt{(2 \pi f)^{2} L_{k} C / 2 N}$ and $\lambda_{p}^{N}=2 \pi / k_{p}^{N}$, respectively, where $N$ is the number of SWCNTs in a bundle.

From (4), we can see that a small $k_{p}^{N}$ leads to large radiation resistance. As a result, the radiation efficiency is improved.

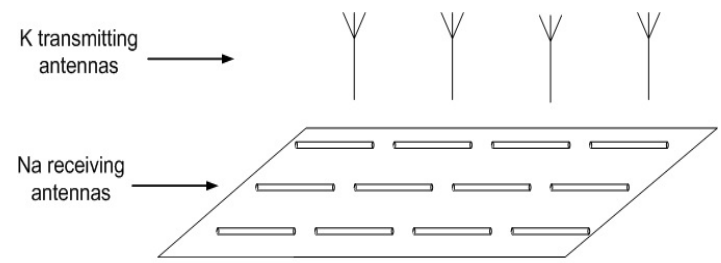

Fig. 2. MIMO system based on SWCNT bundled antennas. There are $N_{a}$ SWCNT bundled antennas used as receiving antennas and $K$ transmitting antennas around the array.

The efficiency of a bundled antenna with $N$ SWCNTs is given by

$$
\xi\left(N, L_{a}\right)=\frac{N^{2}}{N^{2}+B\left(N, L_{a}\right)},
$$

where $B\left(N, L_{a}\right)=\frac{2 R_{c n t} \pi^{2} f^{2} L_{k} C}{k_{0}^{2} \eta \beta\left(k_{p}^{N}, L_{a}\right)}$. As shown in [8], for a fixed $N$, when $L_{a}=\lambda_{p}^{N}$, the antenna efficiency is the highest. Compared with single SWCNT dipole antennas, the simulation results in [11] show that the efficiency of a bundled antenna can increase about $30-40 \mathrm{~dB}$ when the bundle size is 400 .

In fabrication, by changing the catalyst particle size and the growth time, we can control the diameter and the length of SWCNTs, yet it is a challenge to control the bundle size [13]. Denote $\bar{N}$ as the target bundle size, then the real bundle size $N$ obeys truncated normal distribution with the probability density function

$$
\Phi(N)=\frac{1}{\sqrt{(2 \pi)} \sigma} e^{-\frac{(N-\bar{N})^{2}}{2 \sigma^{2}}} \quad N \geq 1,
$$

where $\sigma^{2}$ is the variance of $N$.

\section{Model OF MIMO NeTwORK AND SPECTRAL EFFICIENCY ANALYSIS}

In our model, we use bundled SWCNTs to fabricate receiving dipole antennas. We set the length of the antenna as the full wavelength which has the highest efficiency [8]. The area effectively occupied by each antenna is denoted by $A$, where approximately $A \propto L_{a}^{2}$. For a fixed area $S$, the maximum number of antennas is $N_{a}(\bar{N})=S / A$. Assume that there are $K$ ideal transmitting antennas in this system, where $K$ is in the order of several tens. Thus, we can model the MIMO system based on SWCNT bundled antennas as shown in Fig. 2. For classical metallic dipole antennas, the spectral efficiency depends on signal-to-noise ratio (SNR) and $N_{a}(\bar{N})$. Antenna efficiency is rarely considered since the ohmic resistance of a metallic antenna is much smaller than its radiation resistance, and the efficiency of the antenna is almost unity. However, this is not true for SWCNT antennas as the antenna efficiency is much lower than unity. Besides SNR and $N_{a}(\bar{N})$, antenna efficiency must also be considered when calculating the spectral efficiency of SWCNT antenna systems. From (4) and (7), we can find that increasing the bundle size $\bar{N}$ improves the efficiency of a bundled antenna and hence enhances the spectral efficiency of the system. On the other hand, when $\bar{N}$ increases, $k_{p}^{\bar{N}}$ drops gradually, leading to the rise of $\lambda_{p}^{\bar{N}}$. As a result, each bundled antenna length is larger, and the number of antennas that can be placed in the area $S$ is reduced, which has a negative effect on the spectral efficiency of the system. Therefore, a tradeoff exists between 
$N_{a}(\bar{N})$ and the efficiency of antennas. Next we analyse the model in two scenarios in detail.

a) Large scale MIMO: the array size is relatively large, and the number of antennas $N_{a}(\bar{N})$ increases to hundreds or even thousands. Usually $K$ is much smaller than $N_{a}(\bar{N})$. As the surfaces of the objects in the propagation environment are relatively rough compared to the wavelength in $\mathrm{THz}$, this effect leads to multi-path components. Thus in this study, we assume a Rayleigh fading channel, and the components of the channel matrix $\mathbf{H}$ are independent, identically distributed with zero mean and unit variance [2], although this has not been verified by any measurements to date. When using a zeroforcing $(\mathrm{ZF})$ receiver to detect symbols, the pseudo inverse of $\mathbf{H}$ is needed. However, it is not easy to get the pseudo inverse of a large-size $\mathbf{H}$ directly. Fortunately, from the Law of Large Numbers, we know that the effect of small-scale fading can be averaged out by increasing the number of receiving antennas [2]. From the receiver's perspective, we can simply use the transpose of the matrix instead of its inverse since it is almost true that $\mathbf{H}^{T} \mathbf{H}=\mathbf{I}$ for large size $\mathbf{H}$. The uplink spectral efficiency of the system is lower bounded by

$$
R=\sum_{k=1}^{K} \log _{2}\left(1+S N R_{k} \cdot \xi\left(\bar{N}, \lambda_{p}^{\bar{N}}\right) \cdot\left(N_{a}(\bar{N})-K\right)\right),
$$

where $S N R_{k}$ is the nominal received SNR of the $k$ th user when ideal antennas are used. For this scenario, there exists an optimal $\bar{N}$ that maximizes $R$ given a fixed array area $S$. In fabrication, the real bundle size $N$ is not guaranteed to be identical with the target bundle size $\bar{N}$ which makes the $\xi\left(N, \lambda_{p}^{\bar{N}}\right)$ lower. The expected spectral efficiency can be expressed as

$R=\sum_{k=1}^{K} \int_{1}^{\infty} \log _{2}\left(1+S N R_{k} \cdot \xi\left(N, \lambda_{p}^{\bar{N}}\right) \cdot\left(N_{a}(\bar{N})-K\right)\right) \cdot \Phi(N) d N$.

For the $\mathrm{THz}$ channel, the SNR is affected by transmitting power, spreading and reflection loss, molecular absorption noise, antenna electronic noise and any other additional noise [3]. For SWCNT antennas, however, there are no clear models for the electronic noise in the Terahertz regime. Hence, the realistic values of SNR still remain uncertain.

b) Conventional scale MIMO: the array size is small, and the number of antennas is only several tens. Since the size of $\mathbf{H}$ is not large, it is reasonable to use conventional receivers to detect symbols. When all $K$ users have the same transmitting power, the largest uplink spectral efficiency is achieved for $K \geq N_{a}(\bar{N})$ as

$$
\begin{aligned}
& R=\mathbb{E}_{\lambda}\left[\sum_{m=1}^{N_{a}(\bar{N})} \log _{2}\left(1+S N R \cdot \xi\left(\bar{N}, \lambda_{p}^{\bar{N}}\right) \cdot \lambda_{m}^{2}\right)\right] \\
& \leq N_{a}(\bar{N}) \log _{2}\left(1+S N R \cdot \xi\left(\bar{N}, \lambda_{p}^{\bar{N}}\right) \cdot \mathbb{E}_{\lambda}\left[\lambda_{m}^{2}\right]\right),
\end{aligned}
$$

where $\mathbb{E}_{\lambda}[\cdot]$ is the expectation over $\lambda_{m}, \lambda_{1}, \lambda_{2}, \cdots, \lambda_{N_{a}}$ are the singular values of the channel matrix $\mathbf{H}$, and $S N R$ is the common SNR at each receiving antenna. Since the MIMO channel can be treated as $N_{a}(\bar{N})$ SISO channels [14], the uplink rate increases with $N_{a}(\bar{N})$ and decreases with $\xi\left(N, \lambda_{p}^{\bar{N}}\right)$. For this case, there also exists an optimal $\bar{N}$ that maximizes $R$ given a fixed array area $S$. In this scenario, the spectral efficiency with fabrication variations is modified to

$$
\begin{array}{r}
R=\mathbb{E}_{\lambda}\left[\sum_{m=1}^{N_{a}(\bar{N})} \int_{1}^{\infty} \log _{2}\left(1+S N R \cdot \xi\left(N, \lambda_{p}^{\bar{N}}\right) \cdot \lambda_{m}^{2}\right) \cdot \Phi(N) d N\right] \\
\leq N_{a}(\bar{N}) \int_{1}^{\infty} \log _{2}\left(1+\overline{S N R} \cdot \xi\left(N, \lambda_{p}^{\bar{N}}\right)\right) \cdot \Phi(N) d N
\end{array}
$$

where $\overline{S N R}=S N R \cdot \mathbb{E}_{\lambda}\left[\lambda_{m}^{2}\right]$.

\section{Results}

We consider the system working at frequency $f=2.6 \mathrm{THz}$ which is one of the $\mathrm{THz}$ transmission windows, and in all results, the radius of a single SWCNT is $0.339 \mathrm{~nm}$. The length of SWCNT bundled full wavelength dipole antennas with respect to $\bar{N}$ is shown in Fig. 3(a). For example, when $N$ is 2000 , the optimal antenna length is about 20 $\mu \mathrm{m}$, and the radius of the antenna is $15 \mathrm{~nm}$. At the same frequency, the length of a perfect copper half wavelength dipole antenna is about $60 \mu \mathrm{m}$. This indicates that we can implement nine times the number of antennas in the same area if we use SWCNT bundled antennas instead of copper antennas. Fig. 3(b) shows the number of antennas in an array of $N_{a}(\bar{N})$ versus $\bar{N}$. As can be seen, $N_{a}(\bar{N})$ decreases when the SWCNT's bundle size increases. The relationship between efficiency and the SWCNT bundle size is shown in Fig. 3(c). It can be seen from (7) that when $\bar{N}^{2}$ is much smaller than $B$, the efficiency $\xi\left(\bar{N}, \lambda_{p}^{\bar{N}}\right)$ increases approximately linearly with $\bar{N}^{2}$. As shown in Fig. 3(c), when $\bar{N}$ is 400, the efficiency is $38.8 \mathrm{~dB}$ higher than that of the SWCNT antennas which coincides with the results in [11]. When $\bar{N}$ increases to 2000, the efficiency reaches about 0.3 , which is only $5.2 \mathrm{~dB}$ lower than an ideal antenna. As shown in Fig. 3(b) and (c), we cannot increase the efficiency and the number of antennas simultaneously. Reference [5] gives the efficiency of copper dipole antennas with different diameters. By comparison, the efficiency of SWCNT bundled dipole antennas is more than $10 \mathrm{~dB}$ higher than that of copper dipole antennas of the same diameter. When the diameter gets smaller, the efficiency advantage of using SWCNT bundled antennas becomes even larger. For copper antennas of the same length of SWCNT bundled antennas, the resonant frequency will be much higher than $2.6 \mathrm{THz}$.

In Fig. 4, the solid line shows the spectral efficiency of the large scale MIMO system for different $\bar{N}$. We set the number of transmitting antennas $K=20$, and the antenna area size $S=1 \times 10^{-6} \mathrm{~m}^{2}$. When $\bar{N}$ is less than 1000 , the spectral efficiency increases quickly as $\bar{N}$ gets larger. It indicates that in this region, the efficiency of antennas plays a more important role than the number of antennas in promoting spectral efficiency. From this figure, we can see that there is an optimal value $\bar{N}$ near 2000 which makes the spectral efficiency maximal. When $\bar{N}$ is larger than 2000 , the spectral efficiency reduces slowly with the increase of $\bar{N}$. This implies that the effect of the number of antennas gradually outweighs the efficiency of antennas. The star line shows the theoretical spectral efficiency when there exists fabrication variation. As the standard deviation $\sigma$ increases from $0,0.1,0.3$ to 0.5 , the spectral efficiency decreases obviously. However, the optimal 

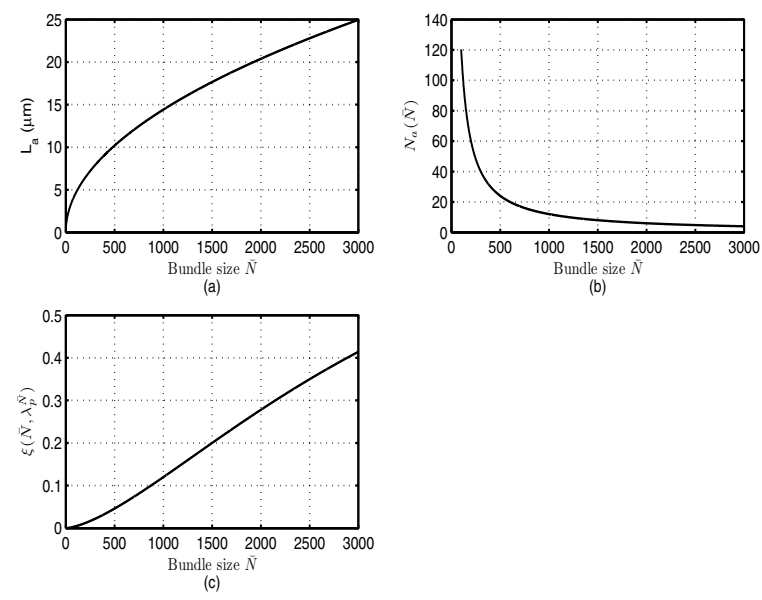

Fig. 3. (a) The length of antennas $L_{a}$. (b) The number of antennas $N_{a}(\bar{N})$. (c) The efficiency of antennas $\xi\left(\bar{N}, \lambda_{p}^{\bar{N}}\right)$. The array size $S=1 \times 10^{-8} \mathrm{~m}^{2}$, and $f=2.6 \mathrm{THz}$.

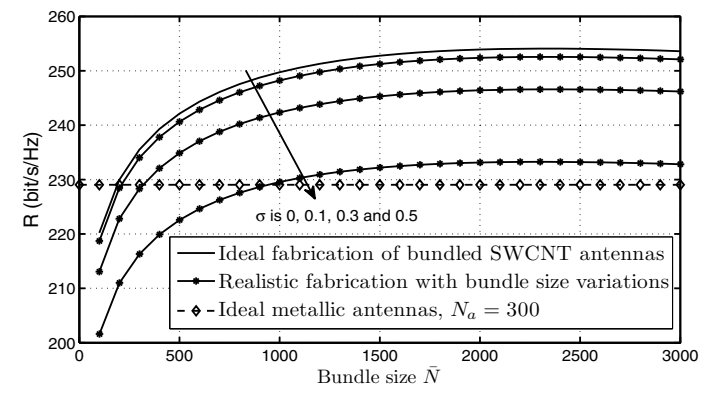

Fig. 4. Uplink spectral efficiency for large scale MIMO, with $S=1 \times$ $10^{-6} \mathrm{~m}^{2}, K=20, S N R_{k}=10 \mathrm{~dB}$ and $f=2.6 \mathrm{THz}$.

$\bar{N}$ is only slightly influenced. As a benchmark, the dashed line in Fig. 4 shows the performance of an ideal metallic antennas based MIMO system of the same $S$. The result indicates that when the fabrication variance is not large, the SWCNT bundled antenna based MIMO system has a higher spectral efficiency (by about 10\%) than systems with ideal metallic antennas.

For the conventional scale MIMO system, the spectral efficiency is shown in Fig. 5. We consider the same parameters as in Fig. 4, but choose the antenna area size as $S=1 \times 10^{-8} \mathrm{~m}^{2}$. These curves show similar behavior as those in Fig. 4. Compared with Fig. 4, the peaks are much more obvious, and the spectral efficiency changes faster as $\bar{N}$ changes. The optimal value $\bar{N}$ is about 800 and when $\bar{N}$ is larger than 800 , the efficiency decreases quickly. Therefore, we can conclude that the spectral efficiency is more sensitive to $\bar{N}$ than under scenario a. The dashed line has the same function as the line in Fig. 4. It shows that for a limited array size, the SWCNT bundled antennas based MIMO system has a higher spectral efficiency than ideal metallic antennas based MIMO systems.

\section{CONCLUSiON}

The SWCNT bundled antenna has many distinctive characteristics because of its unique electrical properties. We have studied the characteristics of SWCNTs and SWCNT bundled antennas and analysed the spectral efficiency of MIMO systems based on SWCNT bundled dipole antennas.

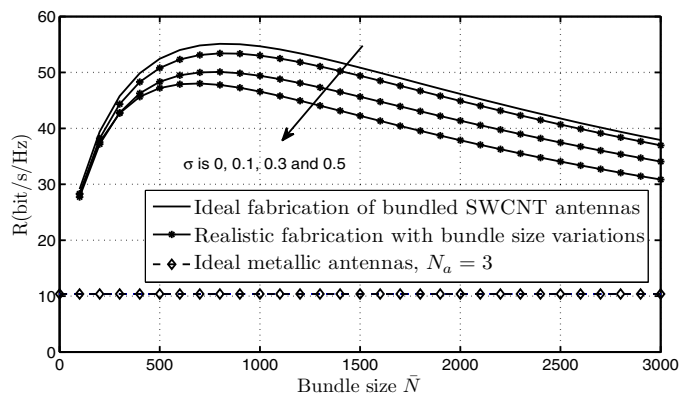

Fig. 5. Uplink spectral efficiency for conventional scale MIMO, with $S=$ $1 \times 10^{-8} \mathrm{~m}^{2}, K=20, \overline{S N R}_{m}=10 \mathrm{~dB}$ and $f=2.6 \mathrm{THz}$.

There is a trade-off between the efficiency of antennas and the number of antennas as a function of bundle size. Under different scenarios, there exists an optimal bundle size which maximizes spectral efficiency. We showed that, for short range communications, the SWCNT bundled antennas based MIMO system has a higher spectral efficiency than ideal metallic antennas based MIMO systems. Furthermore, we investigated the effect of non-uniform bundle size in the fabrication process and showed that such variation can reduce spectral efficiency.

\section{ACKNOWLEDGEMENTS}

The authors would like to express their appreciation to Dr. Tao Lu of the University of Victoria for useful discussions on CNT bundle fabrications.

\section{REFERENCES}

[1] T. L. Marzetta, "Noncooperative cellular wireless with unlimited numbers of base station antennas," IEEE Trans. Wireless Commun., pp. 3590-3600, Nov. 2010.

[2] H. Q. Ngo, E. G. Larsson, and T. L. Marzetta, "Energy and spectral efficiency of very large multiuser MIMO systems," IEEE Trans. Commun., pp. 1436-1449, Apr. 2013

[3] J. M. Jornet and I. F. Akyildiz, "Channel modeling and capacity analysis for electromagnetic wireless nanonetworks in the terahertz band," IEEE Trans. Wireless Commun., pp. 3211-3221, Oct. 2000.

[4] I. F. Akyildiz and J. M. Jornet, "Electromagnetic wireless nanosensor networks," Nano Commun. Networks J., pp. 3-19, Mar. 2010.

[5] G. W. Hanson, "Fundamental transmitting properties of carbon nanotube antennas," IEEE Trans. Antennas Propag., pp. 3426-3435, Nov. 2005.

[6] A. M. Attiya, "Lower frequency limit of carbon nanotube antenna," Prog. Electromagn. Res., pp. 419-433, 2009.

[7] G. W. Hanson, "Current on an infinitely-long carbon nanotube antenna excited by a gap generator," IEEE Trans. Antennas Propag., pp. 76-81, Jan. 2006.

[8] P. J. Burke, S. Li, and Z. Yu, "Quantitative theory of nanowire and nanotube antenna performance," IEEE Trans. Nanotechnol., pp. 314$334,2006$.

[9] C. Rutherglen and P. Burke, "Nanoelectromagnetics: circuit and electromagnetic properties of carbon nanotubes," Small, pp. 884-906, Apr. 2009.

[10] J. J. Plombon, K. P. O'Brien, F. Gstrein, V. M. Dubin, and Y. Jiao, "High-frequency electrical properties of individual and bundled carbon nanotubes," Appl. Phys. Lett., p. 063106, Feb. 2007.

[11] Y. Huang, W. Yin, and Q. Liu, "Performance prediction of carbon nanotube bundle dipole antennas," IEEE Trans. Nanotechnol., pp. 331337, May 2008.

[12] P. J. Burke, "An RF circuit model for carbon nanotubes," IEEE Trans. Nanotechnol., pp. 55-58, Mar. 2003.

[13] Y. Tu, Z. P. Huang, D. Z. Wang, J. G. Wen, and Z. F. Ren, "Growth of alignd carbon nanotubes with controlled site density," Appl. Phys. Lett., pp. 4018-4020, May 2002.

[14] D. Tse and P. Viswanath, Fundamentals of Wireless Communication. Cambridge University Press, 2005. 